\title{
Gaze Perception Triggers Reflexive Visuospatial Orienting
}

\author{
Jon Driver, Greg Davis, and Paola Ricciardelli \\ University College London, UK \\ Polly Kidd, Emma Maxwell, and Simon Baron-Cohen \\ University of Cambridge, UK
}

\begin{abstract}
This paper seeks to bring together two previously separate research traditions: research on spatial orienting within the visual cueing paradigm and research into social cognition, addressing our tendency to attend in the direction that another person looks. Cueing methodologies from mainstream attention research were adapted to test the automaticity of orienting in the direction of seen gaze. Three studies manipulated the direction of gaze in a computerized face, which appeared centrally in a frontal view during a peripheral letter-discrimination task. Experiments 1 and 2 found faster discrimination of peripheral target letters on the side the computerized face gazed towards, even though the seen gaze did not predict target side, and despite participants being asked to ignore the face. This suggests reflexive covert and/or overt orienting in the direction of seen gaze, arising even when the observer has no motivation to orient in this way. Experiment 3 found faster letter discrimination on the side the computerized face gazed towards even when participants knew that target letters were four times as likely on the opposite side. This suggests that orienting can arise in the direction of seen gaze even when counter to intentions. The experiments illustrate that methods from mainstream attention research can be usefully applied to social cognition, and that studies of spatial attention may profit from considering its social function.
\end{abstract}

Requests for reprints should be addressed to Jon Driver, Institute of Cognitive Neuroscience, University College London, Queen Square, London WC1N 3AR, UK. Email: j.driver@ucl.ac.uk

This work was supported by grants from the Human Frontiers Science Program and from the ESRC (UK) to the first author. Our thanks to Vicki Bruce and Steve Langton for helpful discussions of their own independent work on the same topic, and to Marylou Cheal and Ray Klein for comments on the manuscript. 


\section{INTRODUCTION}

This paper seeks to bring together two highly active areas of current research, which have previously been considered quite separately. One area concerns the mechanisms of spatial attention. These have been studied intensively over the last two decades, in many experiments in the spatial cueing tradition instigated by Posner (1978) and others. The second area concerns the mechanisms underlying social cognition. This area has also undergone a tremendous expansion of research activity in recent years (see, e.g. Baron-Cohen, 1995; Brothers; 1990; Premack, 1988). To date, these two areas of research have pursued entirely separate agendas, with entirely different methodologies. Mainstream research on attention has rarely considered orienting in response to stimuli of special social significance, and studies of social attention have not exploited contemporary advances in mainstream attention research. We suggest that these two separate areas of research have a lot to gain from each other when considering certain questions of general psychological interest. We seek to illustrate this for the particular case of the mechanisms underlying "shared attention"; that is, the mechanisms which allow us to judge where other people are attending, and to shift our own attention accordingly.

Brothers (1990) recently set a provocative new agenda for cognitive neuroscience by proposing that the primate brain is primarily a social brain, containing specialized circuits dedicated to social perception and social action. Mainstream attention research has overlooked this emphasis on social function. Brothers' proposal was based upon several lines of argument. First, from an evolutionary perspective, the ability to perceive social relations (e.g. rank, or the current focus of attention of conspecifics) seems highly adaptive. It should allow an animal to benefit maximally from the many potential advantages of a social existence. Second, from a neuropsychological perspective, particular forms of brain damage (such as lesions to the orbitofrontal cortex or to the amygdala; Butter \& Snyder, 1972; Kling \& Brothers, 1992) are already known to lead to quite specific abnormalities in social behaviour, as classically illustrated by the Kluver-Bucy syndrome in monkeys (Kluver \& Bucy, 1938) and by the human case of Phineas Gage (see Damasio, 1995). Third, more recent evidence from neuroscience, using single-cell recording techniques, has revealed the existence of neurons that respond selectively to particular classes of social stimuli. These include faces, hands, eyes and even the apparent direction of attention in seen conspecifics (Brothers, 1995; Bruce, Desimone, \& Gross, 1981; Perrett, Rolls, \& Cann, 1982; Perrett et al., 1990; Perrett \& Emery, 1994).

Baron-Cohen $(1994,1995)$ recently proposed that the brain contains several modules each specialized for different aspects of social existence. Of particular relevance for this paper, he proposed that one such module serves as an "eye-direction detector", identifying the presence of eyes, their direction of 
gaze and any direct eye-contact. There is substantial evidence for a high sensitivity to being looked at across a wide range of species, from reptiles through to primates (Blest, 1957; Burghardt, 1990; Chance, 1967; Ristau, 1990; Scaife, 1976). Human infants spend more time looking at the eyes than at other regions of a face from as early as 2 months of age (Maurer, 1985). In addition to the eye-direction detector, Baron-Cohen also suggested the existence of a "shared attention mechanism" which he argued may be specific to humans or higher primates. This hypothetical mechanism is concerned with whether the self and another agent are both attending to the same object or event, thus allowing for what Bruner (1983) terms "joint attention".

By 14-18 months of age, normal human infants all exhibit joint attention, by means of the protodeclarative pointing gesture (both reception and production thereof), and also by gaze-following (Bates et al., 1979; Bruner, 1983; Scaife \& Bruner, 1975). The exception to this universal development comes from children with the neuropsychiatric condition of autism (Baron-Cohen, 1989; Baron-Cohen, Allen, \& Gilberg, 1992; Baron-Cohen et al., 1996a, b). Baron-Cohen (1995) took the characteristic ontogenesis of shared attention, and its selective impairment in autism, as preliminary evidence for a specialized module for shared attention.

In the present paper, we apply a further traditional criterion for modularity (see Fodor, 1983) to the specific case of shared attention in response to seen gaze; namely, its possible automaticity of operation in adult humans. The classic example of shared attention arises when people orient in the direction of another's gaze (Bruner, 1983; Butterworth, 1991). Here we test whether such orienting arises "automatically", in two specific senses. First, does such shared attention arise even when a person has no particular intention to follow the seen gaze? Second, is shared attention as a consequence of gaze perception automatic in the stronger sense of arising even when one has the express intention of preventing oneself from orienting in the direction of seen gaze? We begin with a brief review of what is currently known about gaze perception, and the shared-attention behaviour that it can trigger.

Many species gaze towards regions of the environment that are currently of particular interest to them, in order to sample these regions with their most sensitive visual receptors. The direction in which other animals or people look can therefore convey considerable information to an observer, by signalling the observed party's current interests. If that party suddenly looks in a specific direction away from the observer, this might signal the location of possible food, of possible danger, of an attractive conspecific, or of a threatening animal (see Byrne \& Whiten, 1991; Menzel \& Halperin, 1975). If the other party suddenly looks towards the observer, this may be an early warning that a sudden attack or some other important interaction is imminent. Indeed, direct gaze is treated as threatening by many species, as shown when captive monkeys display fear responses for gaze directed towards them (e.g. Mendelsohn, Haith, \& 
Goldman-Rakic, 1982; Perrett \& Mistlin, 1990), or when human subjects react similarly to prolonged gaze from a stranger (e.g. Argyle \& Cook, 1976). Of course, mutual gaze can also signal attraction instead of threat, as between lovers (e.g. Rubin, 1970).

As noted earlier, young human infants look more at the eyes than elsewhere in faces (Maurer, 1985). They are also demonstrably sensitive to the direction of seen gaze from around 4 months (Papousek \& Papousek, 1979; Samuels, 1985; Vecera \& Johnson, 1995), often smiling when gazed at (Wolff, 1963). Moreover, the "peekabo" game is a universal social routine with young infants (Bruner, 1983), which involves concealing and then revealing the eyes repeatedly. Adult humans are highly sensitive to the gaze direction of other people, as shown by several psychophysical studies (e.g. Anstis, Mayhew, \& Morley, 1969; Gibson \& Pick, 1962; Watt, 1992). This high sensitivity is lost after brain injury in some prosopagnosic patients (Campbell et al., 1990). Similarly, the sensitivity of macaque monkeys to gaze direction is impaired following lesions to the superior temporal sulcus, a cortical region where single cells have been found to exhibit fine-tuning for the direction of gaze in a seen face (Perrett \& Mistlin, 1990).

Gaze direction is used to regulate various social interactions in humans and other primates, such as dominance confrontations and grooming (Chance, 1967; Van Hooff, 1962), or turn-taking in communication. Bruner (1983) has argued extensively that gaze interactions between adults and preverbal infants form an essential precursor to initial language acquisition. Baldwin (1991) showed that gaze perception plays a role in vocabulary acquisition by toddlers, since the direction in which a speaker looks can indicate the intended referent of unfamiliar words. Based on such findings, Baron-Cohen (1995) has argued that gaze perception forms an essential component of the ability to infer other people's mental states, especially their current focus of attention, their interest and their goals.

Detecting the direction of gaze can lead to joint attention even in infants. Scaife and Bruner (1975), Butterworth (1991) and others have videotaped infants of various ages as they face their mothers, who suddenly divert gaze to look at a particular object in the room. Even at 4 months of age, infants make some eye-movements in the corresponding direction, although there is a developmental progression in their ability then to fixate upon exactly the same object as the mother with just a single saccade (see Corkum \& Moore, 1994; Hood, Willen, \& Driver, 1998). Like other aspects of joint attention, the development of such gaze-following is strikingly abnormal in autism (Baron-Cohen, 1989; Baron-Cohen et al., 1996a; Leekham et al., in press).

Given all this evidence for the importance of gaze perception in determining the direction of normal attention, and its apparent involvement in various pathologies affecting attention, it is very striking that the topic goes quite unmentioned in the extensive literature on visuospatial orienting, within 
mainstream attention research on adult humans (for reviews, see Klein, Kingstone, \& Pontefract 1992; Posner, 1980; Spence \& Driver, 1994). Such research has entirely overlooked Brothers' maxim that the human brain is largely a social brain, and has focused instead on purely asocial situations to date. Experiments in this tradition have typically examined reflexive orienting in response to meaningless but salient events (e.g. abrupt sounds or sudden flashes), or deliberate orienting in response to entirely arbitrary instructions (e.g. expect a target on the left). Such studies have not been concerned with orienting in response to events of particular ecological or social significance, such as sudden changes in the direction of gaze by a conspecific, which may induce shared attention.

Nevertheless, this mainstream orienting literature has successfully developed several powerful "cueing" methods for measuring any spatial orienting that may arise, and for examining its exact nature. The present paper therefore adapts these cueing methods to study the specific case of orienting by adult humans in response to the direction of seen gaze. Our aims in so doing were two-fold: first, to use the established cueing methods to gain further insights into the mechanisms by which gaze perception directs orienting; and, second, to place more social questions about spatial orienting onto the agenda of mainstream attention research, and thus into the realm of the extensive spatial cueing literature.

The cueing method for studying spatial orienting was popularized by Posner $(1978,1980)$ and his colleagues. In a prototypical study, adult subjects are asked to detect visual targets, which may appear on either side of fixation. Their attention can be cued to one side or another before the target appears (e.g. by a brief but uninformative flash on that side, or merely by the instruction that targets are most likely on that side). The robust finding of many such studies is that target detection is often more rapid on the cued side, owing to orienting in that direction.

Variations on this basic cueing methodology have led to two important distinctions. The first is that between "overt" orienting and "covert" orienting. Overt orienting refers to shifts in receptors, such as eye-movements towards the cued side, which will obviously enhance target detection on that side owing to the greater sensitivity of foveal receptors. However, even when no such overt orienting is permitted, target detection can still be faster on the cued side than on the uncued side; this is usually attributed to internal, covert shifts of attention in the cued direction (e.g. Posner, 1978, 1980). Although the overt/covert distinction can be important for a full understanding of the underlying mechanisms responsible for any cueing effect, it is tangential to our main concerns here. Our central goal was to examine the automaticity of any orienting in response to seen gaze. For this purpose, overt and covert orienting are both of interest, and so we had no reason to exclude overt orienting from our experiment by preventing or monitoring eye-movements by the participant. 
The second distinction that has arisen from the cueing paradigm reflects the different types of cue that can induce spatial orienting. When a salient peripheral event acts as the cue (by appearing directly at a possible target location, but without predicting where the target is most likely to appear), the advantage for targets on that side emerges rapidly but is short-lived (e.g. Spence \& Driver, 1994); and may even reverse to become a disadvantage on the cued side at longer delays following the cue (e.g. Posner \& Cohen, 1984). These effects of uninformative but salient peripheral cues seem to be largely reflexive, as the initial advantage on the cued side can be unaffected by emphatic instructions to ignore the cue (Jonides, 1981; Muller \& Rabbitt, 1989), and may arise even if the target is actually more likely on the uncued side (e.g. Spence \& Driver, 1994). Such orienting is therefore termed "exogenous", as it is thought to be under stimulus control (i.e. from without). This contrasts with the orienting that takes place when subjects have an expectancy about where the target will appear (e.g. if a central arrow indicates the most likely target side for that trial; Posner, 1980). Such orienting can be slower to emerge on each trial after the cueing event, but is more durable than the effects of exogenous orienting, being apparent for as long as the expectancy holds. This is termed "endogenous" orienting, since it is under voluntary control (i.e. from within). The distinction between exogenous and endogenous orienting has proved useful in the analysis of both covert attention (e.g. Spence \& Driver, 1994, 1996, 1997) and overt attention (e.g. Henik, Rafal, \& Rhodes, 1995). There is now considerable evidence for several qualitative differences between exogenous and endogenous mechanisms of orienting (e.g. Jonides, 1981; Klein et al., 1992; Muller \& Rabbitt, 1989; Posner \& Cohen, 1984; Spence \& Driver, $1994,1996,1997)$, and it is strongly suspected that different neural substrates are involved (e.g. Rafal, Henik, \& Smith, 1991). Many authors associate exogenous orienting with subcortical structures, in particular the superior colliculus (e.g. Rafal et al., 1991; Spence \& Driver, 1997; Stein \& Meredith, 1993).

The experiments in this paper adapted previous cueing methodologies, and the associated distinctions between various kinds of spatial orienting, to study the specifically social case of orienting in response to the direction of gaze in a seen face. In particular, we sought to determine whether such orienting can arise reflexively, as found for the exogenous orienting observed within standard spatial cueing paradigms, in response to salient but spatially uninformative peripheral events. We first investigated whether orienting in the direction of gaze in a seen face can arise even when that direction is entirely uninformative for the prescribed target task. In our later experiments, we also tested whether such orienting is automatic in the stronger sense of arising even when directly counter to the observer's intentions (Shiffrin \& Schneider, 1977).

Previous, naturalistic studies of spatial orienting in response to seen gaze, within the social-cognition and developmental literatures, suggest that gaze-following is a widespread phenomenon, which can arise spontaneously in 
both human adults and children, and in some other species (e.g. Butterworth, 1991; Menzel \& Halperin, 1975). These findings might seem consistent with the operation of an automatic process. However, the hypothesis of automatic gaze-following cannot be rigorously assessed for these previous experiments, because the direction of seen gaze was either directly relevant to the prescribed task of the subject (as when humans or chimpanzees searched for a hidden reward in the direction correctly indicated by someone else's gaze; Menzel \& Halperin, 1975); or there was absolutely no prescribed task in the observed naturalistic situation (e.g. Butterworth, 1991), thus leaving the degree of intentional involvement in the gazing interaction impossible to determine. Accordingly, while our experiments used fairly naturalistic stimuli (photographed faces), we imposed a somewhat unnatural task, because this more restricted setting allowed us to test directly whether orienting in the direction of another's gaze is an automatic process, in several specific senses.

As discussed earlier, Baron-Cohen (1995) has recently proposed that orienting in response to seen gaze is automatic, in the particular sense of being driven by a specialized Fodorian module. Specialized modules have several defining characteristics according to Fodor (1983), as discussed later. For present purposes, the most critical is that each module is considered to be encapsulated from other processes, and accordingly to operate in an obligatory manner. Our studies provide an initial experimental test of whether this is an appropriate way to characterize orienting in response to seen gaze by adult humans.

\section{EXPERIMENT 1}

Our first study investigated whether the direction of seen gaze, in a photographed face appearing on a computer screen, would induce "exogenous" spatial orienting, in the sense that has been previously defined for uninformative peripheral cues within traditional spatial cueing studies (e.g. Klein et al., 1992; Posner, 1980; Spence \& Driver, 1994). Our method involved aspects from both standard peripheral cueing techniques and from standard central-cueing techniques. A photographed face, looking towards one or other side, served as the cueing event (see Figures 1a and 1b). As with the cue events in previous central-cueing studies (which had typically used arrows pointing towards one or other side; e.g. Posner, 1980), the face cue always appeared at the centre of the screen, at fixation. However, unlike traditional central-arrow cues, the direction in which the central eyes pointed was spatially uninformative about the probable location of the subsequent target, which was equally likely to appear on either side. In this respect, our method followed previous peripheral-cueing studies that used spatially uninformative cues to isolate purely exogenous orienting mechanisms (see Klein et al., 1992; Spence \& Driver, 1994, 1997). The target event which followed our central face-cue was a single letter, appearing to the left or right of the computer screen. Our participants had to judge as 
a.

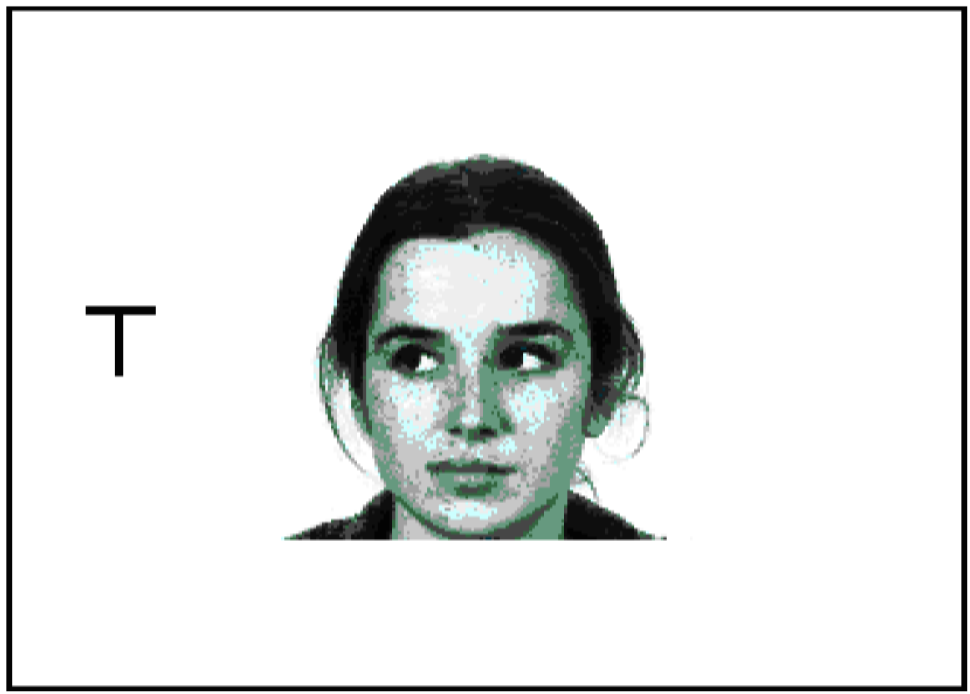

b.

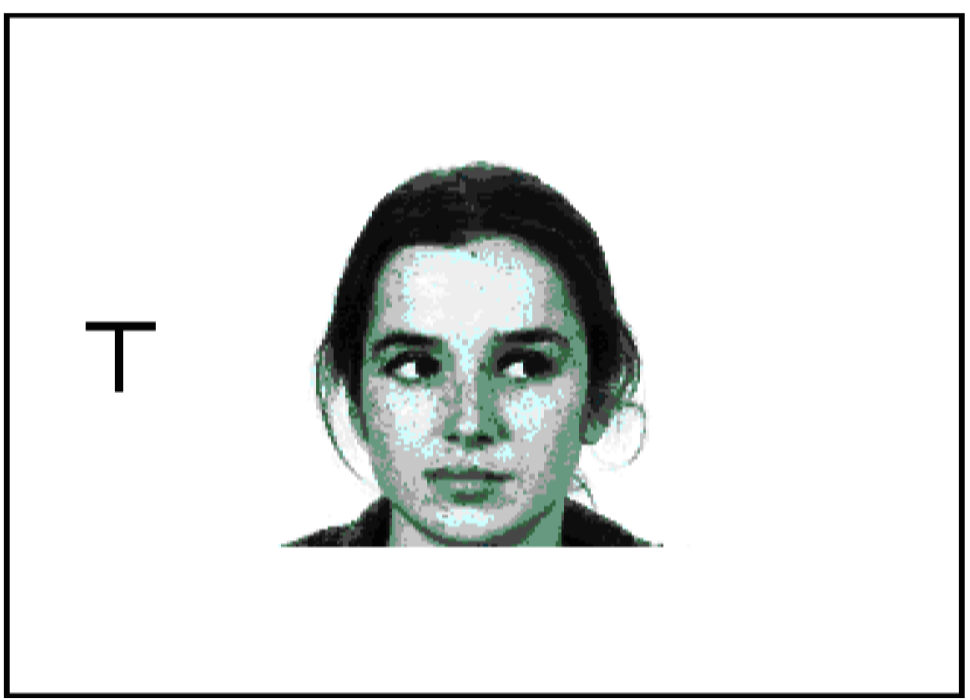

FIG. 1. Reproduction of the digitized face stimuli used as central cues. Panel (a) depicts a spatially congruent trial, where the face gazes towards the side where the target letter (a $\mathrm{T}$ in the illustrated example) subsequently appears. Panel (b) depicts a spatially incongruent trial, where the face gazes away from the subsequent target letter. Note that the two possible face cues were identical in every respect except for the mirror reflection of the eyes. See text for details of the sequence of events on each trial. 
rapidly as possible on each trial whether the letter was a $\mathrm{T}$ or an $\mathrm{L}$, a discrimination which has previously been held to require focused visual attention (e.g. Sagi \& Julesz, 1985; Treisman \& Gelade, 1980).

Note that the face-cue on each trial was totally irrelevant to the prescribed task, since it neither gave any information as to whether the letter to be discriminated would be a $\mathrm{T}$ or an $\mathrm{L}$ on each particular trial, nor indicated whether that letter would appear on the left or the right; this remained equally likely regardless of the direction of the face's gaze. Nevertheless, if orienting in the direction of seen gaze is an automatic process, as Baron-Cohen (1995) has suggested, then it should arise even when the observer has no strategic reason for it to do so. We would then expect target discrimination to be faster for letters appearing on the side that the face gazed towards ("congruent" trials; see Figure1a) than on the side the face gazed away from ("incongruent" trials; see Figure1b), by analogy with the differences that are found between valid and invalid peripheral cues within traditional cueing studies of spatial orienting.

\section{METHODS}

Participants. The nine participants were all undergraduates at Cambridge University, with normal or corrected-to-normal vision, who were naive as to the purpose of the experiment. They all served as unpaid volunteers.

Apparatus and Materials. The experiment was run on a Power Macintosh 7100/8 with a 14-inch Triniton colour monitor, using V-Scope software (Enns, Ochs, \& Rensink, 1990). The participant sat in a dark room, with his or her head stabilized on a chin-rest $60 \mathrm{~cm}$ from the screen. A central asterisk was used as a fixation point and warning signal, subtending $2^{\circ}$. A scanned photograph of a female face was used to produce the cues. It was $6^{\circ}$ in height (see Figures $1 \mathrm{a}$ and $1 \mathrm{~b}$ for scale reproductions of the face) and was displayed using 16 grey levels so that the V-Scope software would run without any blank screens between successive displays. The same basic photograph was used for the gazing-left cue and the gazing-right cue (compare Figures 1a and $1 \mathrm{~b}$ ), with only the eye regions differing between these two cues by a mirror reflection. This was to ensure that no asymmetric properties of the face (wisps of hair, etc.), other than the direction of gaze, could be responsible for any differences in lateral orienting produced by the two cues. The differing eye regions for the two cues were pasted onto the same scanned face photograph, using Adobe Photoshop software, to produce the two face stimuli shown in Figure 1. Target letters were an upper-case $\mathrm{L}$ or $\mathrm{T}$, each subtending $3^{\circ}$ and centred $5^{\circ}$ away from the centre of the screen on one or other side. 
Design. There were two within-subject factors. The first concerned whether the target letter appeared on the side that the central face gazed towards (congruent trials), or on the other side (incongruent trials), which was equally likely. The second factor was the delay on each trial between the appearance of the central face and the subsequent peripheral target letter. This stimulus onset asynchrony (SOA) was equiprobably 100, 300 or $700 \mathrm{msec}$. These SOAs are similar to those used in previous spatial cueing studies with uninformative peripheral cues (e.g. Posner, 1980; Spence \& Driver, 1994, 1997). The typical pattern found with such cues is an advantage for trials on the cued versus uncued side at the $100 \mathrm{msec}$ SOA, which dissipates by around $300 \mathrm{msec}$, and may even reverse to become a disadvantage at the longer SOAs (see Posner \& Cohen, 1984, on the phenomenon of "inhibition of return", which can emerge at longer SOAs following uninformative peripheral cues). Thus, the advantage on the cued side following an uninformative peripheral cue usually emerges very rapidly, but is short-lived. The exact time-course for the predicted cueing effect from gaze direction in the central face for the present study is somewhat hard to anticipate, however. Any advantage on the congruent side might emerge considerably slower than that found after peripheral cues, for two reasons. First, the sudden appearance of a face at the centre of the computer screen, and the associated visual transient, will presumably attract exogenous visual attention to that central location for some time. Second, it will presumably also take some time for the direction of gaze in the face to be encoded, and this process may be somewhat slower than the localization of a salient peripheral cue. Thus, we had no specific prediction for the exact time-course of any cueing effect from gaze in the face. Our expectation was simply that at an advantage for congruent trials should be found at some appropriate SOA, if orienting in response to seen gaze is indeed triggered automatically. Any such effect seemed most likely to emerge at our longer SOAs.

Procedure. Each participant was requested to discriminate the target letter as rapidly and accurately as possible on every trial, by pressing the $\mathrm{H}$-key on the computer keyboard with the index finger of their preferred hand to indicate the letter $\mathrm{T}$, or by pressing the spacebar with the thumb of that hand to indicate the letter L. Thus, the keypress response was in effect an up/down choice of keys. This was used in an effort to reduce the variance that might be produced by any Simon effect (Simon \& Craft, 1970) that could have arisen if a left response had ever been required for a target letter appearing on the right, or vice versa. It was repeatedly emphasized that the central face was entirely irrelevant to the prescribed letter task, and that the direction of its gaze gave absolutely no information about where the target letter would appear on each trial. The only role for the face as far as the participants were concerned was that they were instructed to fixate at the centre of the screen, where the face appeared, throughout each block of trials. 
The sequence of events on each trial was as follows. The fixation asterisk appeared at the centre of the screen for $675 \mathrm{msec}$. This was then replaced by a face-cue, which was equally likely to gaze towards the left or right. After a variable delay, depending on the SOA for that trial $(100,300$ or $700 \mathrm{msec})$, a target letter then appeared on one or other side, remaining present along with the face-cue until the participant's response. This response triggered a feedback signal (a "+" for correct responses, or a " - " for incorrect responses) for 675 msec, and then the whole cycle of events was repeated to produce the next trial. Within each block, congruent and incongruent trials were equiprobable, and were crossed with the three equiprobable SOAs, in an otherwise random sequence. Each participant underwent nine blocks of 60 trials, with the First block being discarded as practice to familiarize them with the target set and with the possible responses. Feedback on mean reaction time (RT) and accuracy was given at the end of each block.

\section{Results}

The median RTs, and associated error rates, were derived for each of the six conditions (congruent/incongruent $\times 3$ SOAs) for every participant. The inter-participant means of these median RTs are shown in Figure 2 (the RT scale of this figure was chosen to allow a direct visual comparison with our subsequent experiments), together with the mean error percentages in parentheses. A two-way, within-subject analysis of variance on the RT data, with the factors of congruency (2) and SOA (3), found a main effect of congruency, $F(1,8)=5.7, p<05$, indicating faster RTs on congruent trials (see Figure 2). A significant main effect of SOA was also found, $F(2,16)=9.6, p<.01$; RT fell as SOA increased, which presumably reflects the standard non spatial temporal warning effect that is seen after any cue event at longer intervals (see Posner, 1978; Spence \& Driver, 1994, 1997). The interaction between congruency and SOA did not approach significance, $F(2,16)=0.4$. Nevertheless, for completeness, we also conducted planned comparisons of congruent and incongruent trials at each individual SOA. We found no reliable effect of spatial congruence at the 100-msec SOA, $F(1,8)=2.5, p=.2$, nor at the $300 \mathrm{msec} \mathrm{SOA}, F(1,8)=0.4$, $p=.6$. However, a reliable spatial congruency effect was found at the 700-msec SOA, $F(1,8)=6, p<.05$.

The error rates were low (see Figure 2 ) and did not vary systematically with congruency or SOA, and showed no signs of any speed-accuracy trade-off. A two-way, within-subject, analysis of variance on the error data found that no effects approached significance, nor was there any interaction (all $F<1$ ).

\section{Discussion}

The direction of gaze by the central face had a reliable effect on performance in the letter-discrimination task, even though the face was totally irrelevant to that 


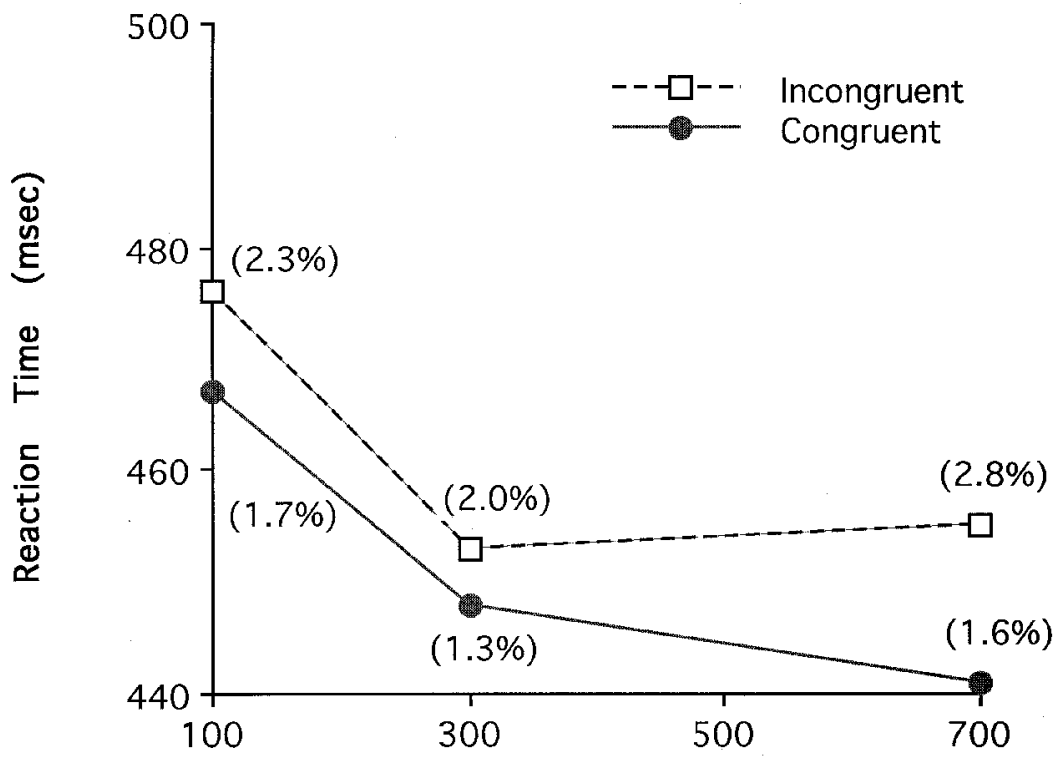

\section{Cue-Target SOA (msec)}

FIG. 2. Inter-participant means of median reaction time in Experiment 1 plotted against cue-target SOA, separately for targets appearing on the congruent versus incongruent side. The numbers in parentheses give error rates for the condition corresponding to the closest RT data point.

task, and provided no information about where the target letter was likely to appear. Participants were reminded that the direction of gaze conveyed no relevant information at the start of every single block (and should in any case have been able to extract this lack of contingency for themselves, since people are extraordinarily sensitive to probabilities in spatial cueing studies; see Spence \& Driver, 1996). Despite the irrelevance of the face's gaze, letter discriminations were still significantly faster on the side that it gazed towards. Note that since a choice discrimination task rather than a simple detection task was used, criterion shifts are unlikely to explain the present cueing effects. Our results suggest that gaze perception triggered a shift of spatial attention (i.e. covert and/or overt orienting) in the corresponding direction, even though there was no strategic motivation for this to happen. In this specific sense, then, orienting in response to the direction of seen gaze can apparently arise "automatically".

The congruence effect on RTs did not interact significantly with SOA in the overall analysis of variance. However, the planned comparisons at individual SOAs showed that the advantage when the central face gazed towards the subsequent target side was most reliable at the 700-msec SOA. This time-course 
for the cueing effect is very different to that found in traditional cueing experiments with uninformative peripheral cues, such as sudden flashes or beeps, where the effects of exogenous spatial orienting emerge at very short SOAs (100 msec or less), and dissipate or reverse rapidly soon after (see Posner \& Cohen, 1984; Spence \& Driver, 1994, 1997). As mentioned earlier, however, the present cueing effect from seen gaze might be expected to emerge more slowly than for standard peripheral cues. It will presumably take some time for the direction of the face's gaze to be encoded, and this may well take longer than coding the location of a salient peripheral cue. On the other hand, it seems unlikely that merely extracting gaze-direction could take as long as the full 700 msec that was allowed at our longest SOA. Such a slow rate of gaze perception seems implausible if it is to serve the rapid early warning purposes in ecological situations that we alluded to in the Introduction. An alternative reason for the relatively delayed time-course of the present cueing effect is the sudden onset of the face-cue, appearing from nowhere at the centre of the, screen, which produces a salient visual transient that should itself draw exogenous attention to the central location. This could delay any subsequent shift of exogenous attention in the direction of the face's gaze. Our second experiment addressed this possibility directly, by substantially reducing the visual transient produced at the centre of the screen by the face-cue.

\section{EXPERIMENT 2}

The next study followed the method of Experiment 1 with just one exception. Participants were now given considerable time to process the face, and to recover from its sudden onset at the centre of the screen, before any eye information was presented. This was achieved in the following way. After the fixation asterisk began each trial, a central face appeared with its eyes occluded by uniform grey regions that approximately matched the average face tone; this stimulus was created by superimposing the grey regions on the scanned face within Adobe Photoshop. It was presented like this (see Figure 3, frame 2) until $900 \mathrm{msec}$ had elapsed. Only after this were the occluding grey regions removed to reveal the eyes (see Figure 3, frame 3). The cue-target SOAs of 100, 300 or $700 \mathrm{msec}$ were again measured from the point of time where the eyes appeared. The new sequence of events within each trial (see Figure 3) gave the impression of a face appearing with eyes closed, with the eyes then blinking open to look towards one side before the appearance of the target letter.

We expected to replicate the advantage for letter discrimination on congruent trials that was found in Experiment 1. If the atypically slow time-course of that cueing effect had been in part due to attention being captured by the sudden appearance of a face at the centre of the screen, we expected that the effect should now become more robust at shorter SOAs, since the new procedure gave 


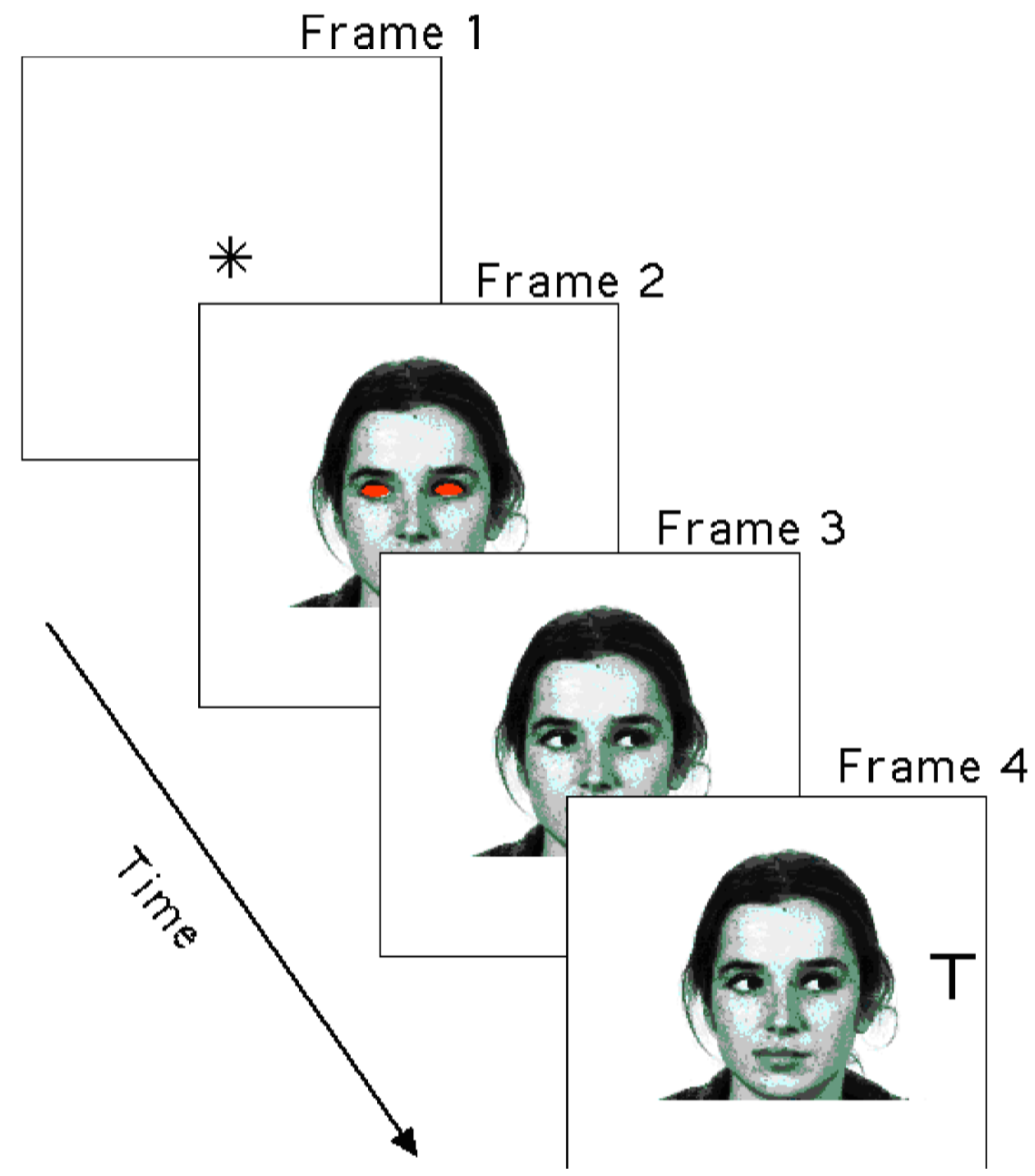

FIG. 3. Schematic depiction of a typical sequence of events within one trial from Experiment 2, with successive events running from top-left to bottom-right in the figure. As in Experiment 1, each trial began with a central asterisk to serve as a fixation point and warning signal (Frame 1). Then, $675 \mathrm{msec}$ later, the face appeared, with eyes appearing "closed" due to the superimposed grey patches (Frame 2). After a further $900 \mathrm{msec}$, the deviated gaze of the face was revealed (Frame 3). Finally, after a variable delay depending on cue-target SOA, the target letter appeared on one side (Frame 4). 
participants $900 \mathrm{msec}$ to recover from the sudden onset of the central face, before the onset of the eyes.

\section{Methods}

The methods were identical to Experiment 1, except for the insertion of a central face with eyes occluded for $900 \mathrm{msec}$ at the start of each trial (see Figure 3, frame 2). The eight new participants were Cambridge University undergraduates with normal vision.

\section{Results}

Figure 4 shows the inter-participant means of median RTs for each condition, together with the mean error percentages in parentheses. A two-way, within-subject analysis of variance on RTs [congruency (2) x SOA (3)] found a main effect of congruency once again, $F(1,7)=35.5, p<.001$, with faster RTs on congruent trials. A significant effect of SOA was also found, $F(2,14)=11.4$, $p<.001$, with shorter RTs at longer SOAs as before. The interaction between congruency and SOA was now significant, $F(2,14)=5.2, p<.05$. As can be

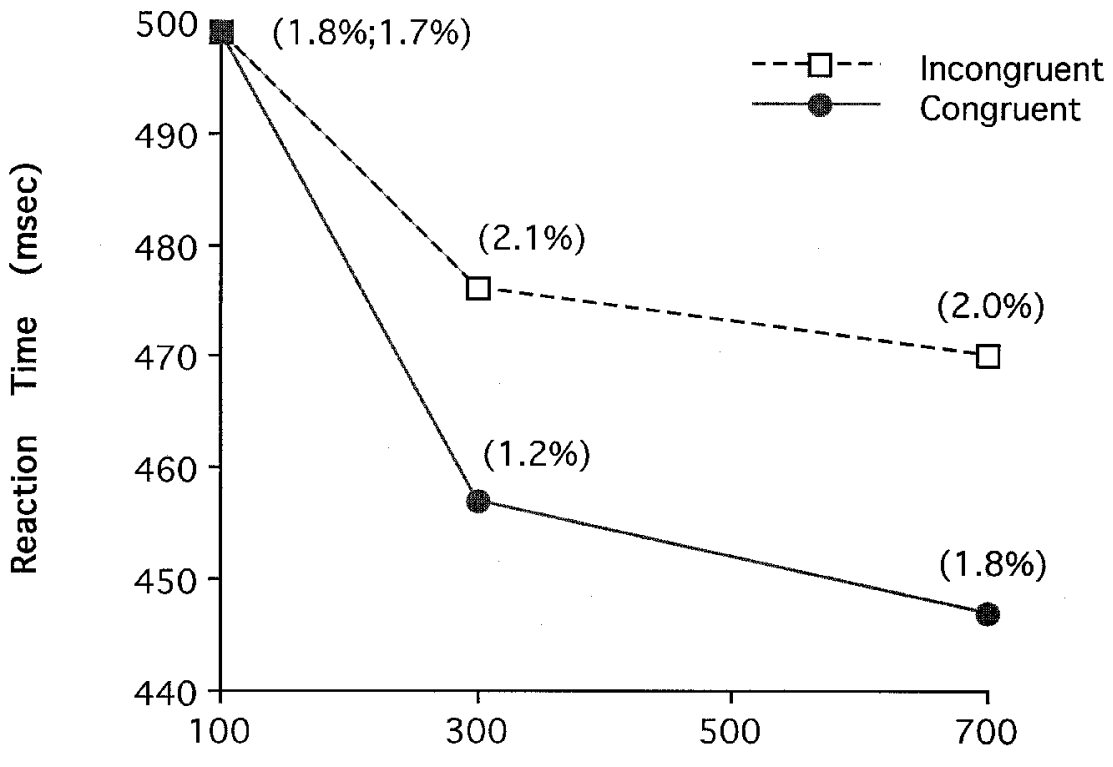

\section{Cue-Target SOA (msec)}

FIG. 4. Inter-participant means of median reaction time in Experiment 2, plotted against cue-target SOA, separately for targets appearing on the congruent versus incongruent side. The numbers in parentheses give error rates for the condition corresponding to the closest RT data point. Where two RT data points overlap, the error rate for the incongruent condition is given first. 
seen in Figure 4, congruency had no effect at the shortest SOA of $100 \mathrm{msec}$, but led to faster RTs on congruent trials at the two longer SOAs. This was confirmed by planned comparisons; these found no effect of congruency at the SOA of $100 \mathrm{msec}, F(1,7)=0.001, p<.98$, but significant congruency effects at both the 300-msec SOA, $F(1,7)=7.1, p=.03$, and the $700 \mathrm{msec}$ SOA, $F(1,7)=52.9, p<.0001$.

As before, the error rates were low and did not vary systematically with congruency condition or SOA, nor showed any signs of speed-accuracy trade-offs. No terms approached significance in an omnibus analysis of variance on the error data.

\section{Discussion}

Experiment 2 replicated the basic cueing effect of Experiment 1, from the uninformative gaze direction of a central face, but this effect was now observed in a more robust fashion (compare Figure 4 and Figure 2). The cues now comprised eyes which suddenly opened in a central face that was already present. This was implemented in an effort to reduce possible attention capture by the central face when it appeared suddenly from nowhere, as had been the case in Experiment 1. In these new circumstances, the advantage for targets in the direction of seen gaze emerged reliably by $300 \mathrm{msec}$ following the appearance of the gazing eyes (see Figure 4, 300-msec SOA data). Nevertheless, the spatial cueing effect was still most pronounced, both numerically and in terms of significance levels, at the longer 700-msec cue-target SOA, suggesting that the time-course of spatial orienting in response to uninformative seen gaze is temporally extended or delayed, as compared with that typically induced by uninformative peripheral cues (cf. Posner \& Cohen, 1994; Spence \& Driver, 1994, 1997).

There was no indication of any reversal in the sign of the spatial cueing effect for the 700-msec SOA as compared with the 300-msec SOA. That is, there was no evidence of the inhibition of return (IOR) phenomenon that can be found at longer SOAs after peripheral uninformative cues, whereby spatially congruent cues start to produce slower responses than spatially incongruent cues, at longer delays (see Posner \& Cohen, 1984). However, IOR is not always apparent in discrimination tasks like the letter judgement required here. being found most reliably in simple detection tasks (see Klein \& Taylor, 1994; Spence \& Driver, 1994, 1997; but see also Lumanez et al., 1997, for a demonstration that IOR sometimes can be found in discrimination tasks). The absence of any IOR in the present study might just be one further example of this particular effect proving elusive in speeded discrimination tasks. Alternatively, its absence might be due to our use of gazing-face cues. Even though our gaze cues were spatially uninformative, they might in some respects behave more like the informative central cues (arrows, etc.) used in previous spatial cueing studies, than like uninformative peripheral cues. Previous studies with non-social cues 
found that IOR is typically associated with peripheral rather than central cues (Klein \& Taylor, 1994; Spence \& Driver, 1997).

When taken together, the prolonged time-course of the present gaze-cueing effect, plus the absence of any IOR, resembles the pattern traditionally produced by informative central cues more closely than that associated with uninformative peripheral cues, in studies with non-social stimuli (arrows or flashes, etc.) rather than faces. However, the most important point for present purposes is simply that the direction of central gaze can produce robust positive spatial cueing effects (rather than negative IOR); and that this cueing effect from the direction of seen gaze emerges relatively fast (within $300 \mathrm{msec}$ ), provided the face was previously visible.

Experiments 1 and 2 demonstrate that seen gaze can induce spatial orienting in adult observers even when the direction of seen gaze is entirely irrelevant to the prescribed task, and thus gives no strategic motivation for any orientingThis seems consistent with orienting in response to seen gaze being a fairly "automatic" process, as suggested by Baron-Cohen's (1995) proposal that shared attention based on gaze perception reflects the obligatory operation of specialized Fodorian modules. Our final experiment tested a more stringent criterion for automaticity (Shiffrin \& Schneider, 1977). Experiments 1 and 2 showed that orienting in response to seen gaze can arise when the perceived gaze is irrelevant to the prescribed task; that is, when the observer has no particular wish to orient in either direction. Experiment 3 tested whether orienting in the direction of seen gaze can arise even when it directly opposes current intentions (i.e. when the observer has the express intention of orienting in the opposite direction, because he or she expects the target to appear on that side).

\section{EXPERIMENT 3}

In the final study, the target was now four times as likely to appear on the side away from where the central face gazed (e.g. Figure 1b). than to appear on the side that the face gazed towards (e.g. Figure 1a). Participants were reminded of these probabilities at the beginning of every block, and in any case had ample opportunity to discover the negative contingency between direction of gaze and likely target side for themselves, within the practice block and subsequent blocks. This should provide considerable motivation for trying to shift attention in the direction that the face gazed away from on each trial; moreover, the participants were explicitly instructed to do this. However, if people cannot completely suppress an automatic tendency to orient in the direction of seen gaze, we would expect that letter discrimination might still show an advantage on the side that the face gazed towards, even though the target letter was now actually four times as likely to appear on the opposite side.

The logic of this study is analogous to previous studies on the automaticity of exogenous spatial orienting in response to meaningless but salient peripheral 
cues. As discussed earlier, such peripheral cues can produce spatial orienting effects even when uninformative about the likely target location. Of more relevance for the present study, peripheral cues can also attract spatial attention towards them even when counter-informative; that is, when targets are more likely on the side opposite the peripheral cue (e.g. Spence \& Driver, 1994). The typical result with such counter-informative peripheral cues is that attention is first exogenously "pulled" to the location of the peripheral event, enhancing target judgements there at short SOAs. Then, as time progresses after the cue, participants are gradually able to "push" their attention endogenously to the other side, where they expect the target to appear, so a performance advantage gradually emerges for that side versus the peripherally cued side that showed the initial advantage. This pattern of results following counter-informative peripheral cues has been taken as strong evidence for the automatic nature of exogenous orienting; salient events evidently pull attention towards them initially, even when the person wishes to attend elsewhere.

Our final experiment tested whether orienting in response to seen gaze is likewise automatic, in this strong sense of arising initially even when counter to intentions. If so, we would expect letter discrimination to be faster on the side that the central face gazes towards (at relatively early cue-target intervals, such as the 300-msec SOA) even though participants strongly expected the target to appear on the opposite side. At longer cue-target intervals (e.g. the present $70 \mathrm{msec}$ SOA), participants may have sufficient time to succeed in "pushing" their attention endogenously over to the expected side, as found at long delays after counter-informative peripheral cues (Spence \& Driver, 1994). If so, any initial advantage for the side that the face gazes towards at shorter SOAs should disappear at longer SOAs, or even reverse to become a disadvantage on that side.

\section{Methods}

This experiment was exactly like Experiment 2 except for a change in the probability of particular conditions. Trials with the target letter appearing on the side that the face gazed away from (Figure 1b) were now four times as likely as trials where the face gazed towards the subsequent target (Figure 1a). Accordingly, the former type of trial is now referred to as "expected" rather than "incongruent", and likewise the previous "congruent" trials are now referred to as "unexpected". The three cue-target SOAs, measured as in Experiment 2 from the onset of the eyes in the pre-exposed face, were again 100, 300 and 700 msec; and the six possible conditions were intermingled in a random sequence for each participant, with the appropriate proportions. The 10 new participants were Cambridge undergraduates with normal vision. 


\section{Results}

Figure 5 shows the inter-participant mean RTs for each condition, together with the mean error percentages in parentheses. Untrimmed mean rather than median RTs were now computed for each participant, as there were more trials in the expected condition than in the unexpected condition owing to our probability manipulation, and Miller (1988) has shown that median RTs can produce spurious differences between conditions in such circumstances. A two-way, within-subject analysis of variance on mean RTs [expectancy (2) x SOA (3)] found no overall effect of the target letter appearing on the expected side (which the face gazed away from) versus the unexpected side (which the face gazed towards), $F(1,9)=0.59$. A significant effect of SOA was found, $F(2,18)=24.9 \cdot p<.001$, with shorter RTs at longer SOAs as before. Critically, the interaction between expected/unexpected side and SOA was significant, $F(2,18)=6.93, p<.01$. As can be seen in Figure 5, RTs were faster for targets on the unexpected side (i.e. in the direction that the central face gazed towards) at the 300-msec SOA, but this pattern appeared to reverse at the 700-msec SOA, with RTs becoming numerically faster on the side where targets could be expected (which the face gazed away from). Planned comparisons found a

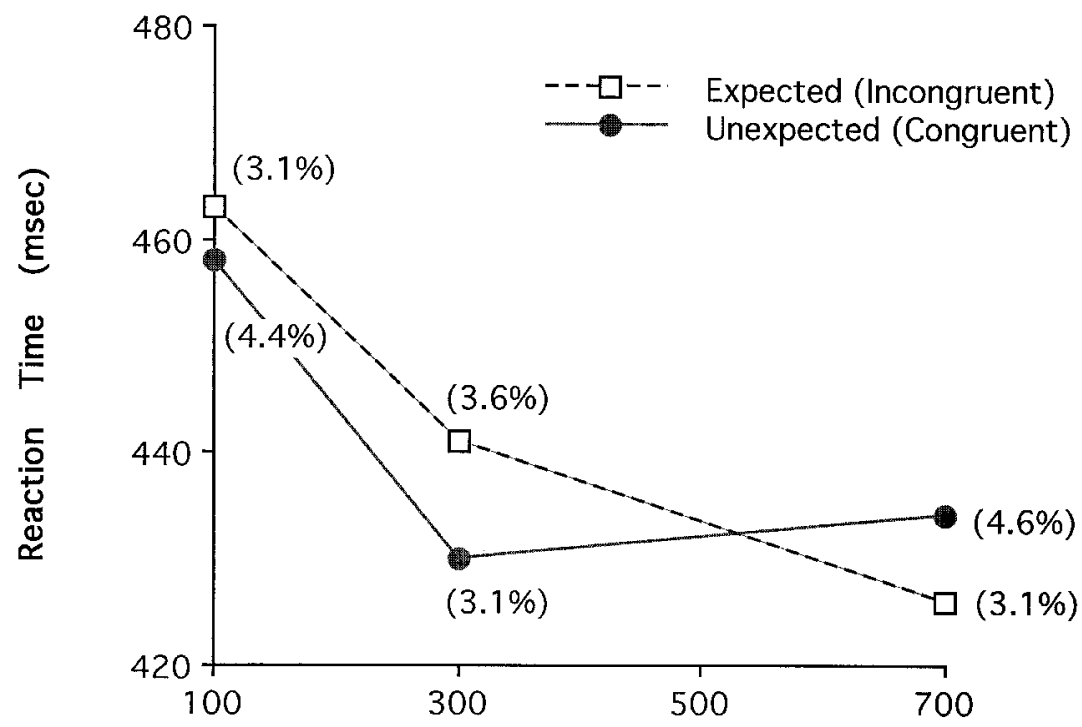

\section{Cue-Target SOA (msec)}

FIG. 5. Inter-participant mean reaction times in Experiment 3, plotted against cue-target SOA, separately for targets appearing on the expected (spatially incongruent) versus unexpected (spatially congruent) side. The numbers in parentheses give error rates for the condition corresponding to the closest RT data point. 
reliable advantage for targets on the unexpected (spatially congruent) side at the 300-msec SOA, $F(1,9)=7.5, p<.03$ ), a trend towards an advantage for targets on the other expected (spatially incongruent) side at the 700-msec SOA, $F(1,9)=3.4, p<.1$, and no effect of target side at the $100-\mathrm{msec}$ SOA, $F(1,9)=1.2, p=.3$.

As before, the error rates were low and did not vary systematically with spatial condition, nor against SOA. A two-way, within-subject analysis of variance on the error data found that no effects approached significance; nor was there any interaction (all $\mathrm{F}<1$ ). Note, however, that any numerical trends were in agreement with the RT results.

Experiments 2 and 3 had identical methods except for the change in probable target location (equally likely on either side in Experiment 2, but four times more likely on the side that the central face gazed away from than on the side that it gazed towards in Experiment 3). As can be seen by comparing Figures 4 and 5, this manipulation of event probabilities had a substantial impact on the results, producing the "crossover" that is apparent in the data at the right of Figure 5 only when targets could be expected on the side that the face gazed away from. We conducted a further between-experiment analysis to examine the reliability of this change in outcome more closely. For the purposes of this analysis, we subtracted each participant's mean RT for trials where the central face gazed towards the subsequent target letter, from the mean RT at the same SOA for trials where the face gazed away from the subsequent target. This subtraction yields a cueing measure, summarizing any advantage for targets on the side that the central face gazed towards. The average cueing effects of this kind for Experiments 2 and 3 are plotted for comparison in Figure 6, separately for each SOA (and with standard error bars indicating variability for each effect).

Note that the cueing advantage for the side that the central face gazed towards is maintained across the 300- and 700-msec SOAs for Experiment 2, but disappears (in fact, numerically reversing to become a disadvantage for targets on that side) at the 700-msec SOA in Experiment 3. To determine the reliability of this difference in the pattern of results, we conducted a two-way, mixed analysis of variance on the cueing measure yielded by the subtractions described above. The between-subject factor was Experiment 2 versus Experiment 3, and the within-subject factor was cue-target SOA. This analysis of variance on cueing effects found main effects of experiment, $F(1,16)=8.1$, $p=.01$, and of SOA, $F(2,32)=3.7, p<.05$. More importantly, there was also a highly reliable interaction, $F(2,32)=8.4, p=.001$, confirming the different temporal pattern of cueing effects in the two experiments. Planned comparisons showed no significant effect of experiment at the 100-msec SOA, $F(1,48)=0.6, p=.4$ or at the $300 \mathrm{msec} \operatorname{SOA} F(1,48)=1.6, p=.2$. However, a highly significant difference between the cueing effect in the two experiments was found at the $70-\mathrm{msec}$ SOA, $F(1,48)=22.8, p<.0001$. Thus, this analysis shows that the change in event probabilities between Experiments 2 and 3 did 


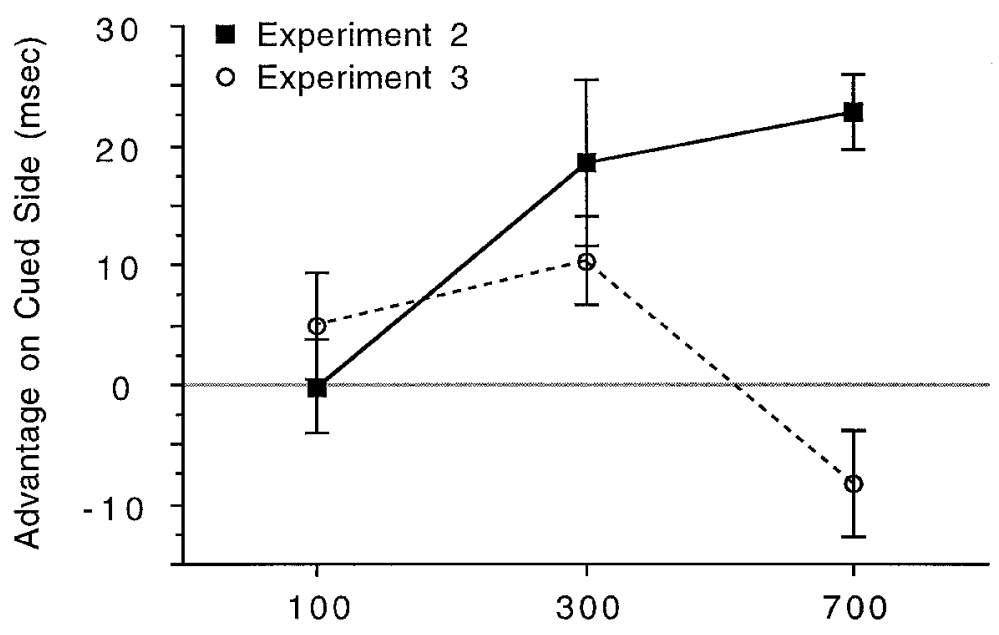

Cue-Target SOA (msec)

FIG. 6. Mean advantage (incongruent RT minus congruent RT) for targets on the side which the computerized face gazed towards, with standard error bars, for Experiment 2 versus Experiment 3 as a function of cue-target SOA.

not significantly alter the cueing effects induced by the gaze of the central face at the 100-msec SOA (where there were no reliable cueing effects), or at the 300-msec SOA (where targets were responded to reliably faster on the side that the face gazed towards). However, by 700-msec after presentation of the cue, the cueing effect was significantly reduced (and, indeed, was reversed in sign) when participants could develop the expectancy that the target was most likely to appear on the side that the face gazed away from (i.e. in Experiment 3 as compared with Experiment 2).

\section{Discussion}

The target letter was now four times as likely to appear on the side that the face gazed away from. Nevertheless, our usual cueing effect-namely an advantage for target letters on the side that the face gazed towards-was replicated once more at the 300-msec SOA. Indeed, the between-experiment comparison with Experiment 2 confirmed that the cueing effect at this interval was not significantly affected by the substantial change in event probabilities. In contrast, by the time the target appeared at the longer SOA of 700-msec, participants' expectancies that the target was more likely to appear on the spatially incongruent side (which the face gazed away from) began to affect performance. The cueing effect at this cue-target interval was significantly reduced in comparison with Experiment 2 (where no spatial expectancy had been possible) and, 
indeed, was numerically reversed in sign. This suggests that participants in Experiment 3 were trying to attend to the side where targets were most likely (i.e. the side which the central face gazed away from). The persistence of our usual cueing effect at the 300-msec S0A, producing an advantage for targets on the side that the face gazed towards, suggests that orienting in the direction of seen gaze can be an automatic process, in the strong sense of arising even when counter to a person's current intentions.

\section{GENERAL DISCUSSION}

The main theme of this paper is that the study of spatial orienting within mainstream attention research may be enriched by considering important social aspects of attention; and that, conversely, research on social cognition might usefully exploit some of the methodological and theoretical advances within mainstream attention research. We hope that our experiments illustrate that these two traditionally separate areas of research can be fruitfully brought together. In our three experiments, we adapted traditional spatial cueing methods, developed within mainstream attention research, to address specific empirical questions concerning "shared attention", a major topic in research on social cognition. In particular, we investigated whether it is appropriate to characterize orienting in the direction of seen gaze as "automatic" in any sense. Folk psychology often invokes a powerful influence from the gaze of others, and a particularly strong tendency to look where others look. Naturalistic experiments in developmental psychology (e.g. Bruner, 1983; Moore \& Dunham, 1994) have confirmed that a tendency for spontaneous gaze-following does indeed exist; and the recent theory of Baron-Cohen (1995) explicitly proposes that gaze perception, and consequent gaze-following, operates in an obligatory manner, under the control of specialized Fodorian modules. However, before our study, there had been no attempt to test the automaticity of gaze-following in any rigorous empirical fashion, with operationalized definitions of what constitutes evidence for or against automaticity.

The most appropriate definition for "automatic" processes has been much debated in cognitive psychology over the last two decades (e.g. Bargh, 1992; Carr, 1992; Logan, 1992; Shiffrin \& Schneider, 1977), leading to an increasing realization that the term can have several subtly different meanings. We examined whether orienting in the direction of seen gaze is automatic in two very specific senses. Our first question was whether such orienting can arise when the observer has no motivation to shift attention in the direction of seen gaze. Our results suggest that this is indeed the case. Experiment 1 found faster letter discrimination on the side that a central face gazed towards, despite the fact that the target letter as just as likely to appear on the opposite side, and even though the adult participants were fully aware of these probabilities, which applied for several hundred trials. Experiment 2 replicated this effect and found that it 
became reliable at shorter intervals following the deviated gaze stimulus if the central face initially appeared with eyes "closed", and only subsequently gazed to one side. (In Experiment 1, the central face had abruptly appeared simultaneously with the onset of its deviated gaze. In this situation, the face presumably attracts considerable attention to itself when first appearing, in a manner that could delay any lateral shift of attention that its gaze might trigger.) Experiments 1 and 2 thus confirm that the direction of seen gaze can trigger corresponding spatial orienting even when known to be uninformative; gazefollowing is thus "automatic" in this specific sense.

Our second question was whether orienting in the direction of seen gaze might also be automatic in a stronger sense; namely. arising even when the observer tries to orient in the opposite direction, and thus operating even when counter to express intentions (Shiffrin \& Schneider, 1977). Our results suggest that shared attention based on gaze perception-looking where another face looks-may also pass this more stringent criterion for automaticity. Experiment 3 found that, shortly after the central face gazed towards one side, letter discrimination remained faster on that side even though our adult participants knew that the target was in fact four times as likely to appear on the other side. Letter discrimination did not start to become faster on the side where the target could be expected (as compared with Experiment 2, where no expectancy was possible) until our longest SOA, where $700 \mathrm{msec}$ had elapsed since the eyes in the central face first deviated towards the other side (see Figure 6).

These results have several implications for mainstream attention research, for the study of social cognition and for future attempts to bring these two areas together in a fruitful manner. First, our experiments provide clear empirical support for many previous informal claims that orienting in the direction of seen gaze may be reflexive. As described above, we have confirmed this for two specific operationalized definitions of automaticity. It will be important to test whether the orienting that we have identified here will also pass the many additional criteria for automaticity that have been proposed in cognitive psychology. As noted in several recent papers (Bargh, 1992; Carr, 1992; Logan, 1992), these various criteria are logically separable, and so should each be considered in their own right by appropriate empirical tests. An incomplete but illustrative list of some of the criteria that have been proposed for the "automaticity" of any process would include the following: operating without intention (cf. Experiments 1 and 2); operating contrary to intention (cf. Experiment 3); independent of set-size; unconscious; innate; highly practised; informationally encapsulated; cognitively impenetrable; modularized; and dependent on dedicated neural systems (Bargh, 1992; Carr, 1992; Fodor, 1983; Logan, 1992; Shiffrin \& Schneider, 1977). Our data clearly speak to some of these criteria more directly than others. We have shown that orienting in the direction of seen gaze can arise when observers have no motivation for responding in this way (Experiments 1 and 2). Moreover, such orienting can 
arise even when people have good reason to orient in the opposite direction (Experiment 3), and where it can be shown that they were trying to do so (as by our comparison of Experiment 3 with Experiment 2).

Our results appear broadly compatible with Baron-Cohen's (1995) notion of specialized mechanisms for orienting in the direction of a conspecific's gaze, and fit his claim that these mechanisms may operate in the mandatory fashion of Fodorian modules, arising "automatically" in the specific senses that we have emphasized. However, it remains for future research to determine whether the orienting that we have documented reflects specialized modules with all the other characteristics that Fodor first proposed. For instance, our data are entirely silent on the issue of whether gaze-following reflects innately specified mechanisms, as Fodor (1983) suggested for his proposed modules, or instead results from extensive social experience. Such issues are hard to address for participants like our own, each of whom presumably came into our experiments with around 20 years of experience that seen gaze can often be predictive of events in the corresponding direction.

Most spatial cueing studies within the mainstream attention literature have not considered whether learning processes, operating over a substantial timespan, could be involved in some of the spatial orienting effects which they have uncovered. For instance, even the well-known effects following uninformative peripheral visual cues, as studied by Posner (1980) and others, might conceivably arise as a result of long-term learning that abrupt peripheral transients are often followed by important events at the same location in the real world. A recent study by Lambert and Sumich (1996) provides a demonstration that learned associations between cue events and the subsequent position of target events can produce reliable spatial cueing effects, even when participants are unaware of the effective contingencies (in their case, arbitrary pairings between word categories and the side of subsequent target probes). The spatial cueing from seen gaze that we have identified here might operate in such a fashion, as the result of extensive experience within social settings where seen gaze direction can often predict the location of important events. The inability to override this association within the context of our experiments, over hundreds of trials, might then arise simply because the tendency to orient in the direction of seen gaze is so heavily overlearned that it cannot easily be suppressed at will, nor unlearned within just one hour. Alternatively, the orienting that we observed might conceivably involve some innate mechanisms specific to gaze perception, as Baron-Cohen (1995) implied. Note that, regardless of which explanation is correct, our experiments would still demonstrate orienting in response to seen gaze for adult observers, which is automatic in the two senses we have described.

Experiments with very young infants may prove more decisive on the learned/innate issue. Naturalistic studies of gaze-following in healthy babies have suggested that it is most reliably found from around 10-12 months of age 
(e.g. Corkum \& Moore, 1995; Scaife \& Bruner, 1975). There have been some suggestions that shared-attention behaviour can occasionally be seen in much younger infants (e.g. Butterworth, 1991; Butterworth \& Jarrett, 1991; Muir, Hains, Cao, \& D'Entremont, 1996), but these all arose from studies involving turns of an adult's head as the trigger event, rather than deviations in seen gaze alone, as in our study. It is usually argued that cues from the eyes alone are only effective in triggering attention shifts by older children (see Moore \& Dunham, 1995). However, a recent study by Hood et al. (1998) adapted the present spatial cueing method for babies, and found that even 3-month old infants oriented faster to a peripheral event if an adult's eyes (in a computerized face) had just gazed in its direction, without any head turn. Hood et al. argue that traditional, naturalistic paradigms for measuring shared attention may underestimate infants' true capacities for interpreting seen gaze, and for shifting their own attention accordingly, because young infants tend to get "locked" onto interesting central stimuli, such as the adult's face, which they initially fixate in standard shared-attention tests (and perhaps also because they have difficulty in orienting laterally without a peripheral trigger stimulus). Hood et al. circumvented both these potential difficulties for young babies by removing a computerized central face after its gaze had deviated to one side (so that the babies could not remained locked onto the face), and then measuring the latency and accuracy of responses to peripheral probes presented equiprobably on either side, within a cueing paradigm that was otherwise very similar to our own. Further studies with this method may reveal orienting in response to seen gaze by even younger babies.

It is interesting to note the similarities (and differences) between the spatial cueing caused by seen gaze in the present studies and that found following uninformative peripheral events in previous non-social studies of exogenous spatial orienting (e.g. Jonides, 1981; Posner, 1980; Spence \& Driver, 1994, 1997). The direction of seen gaze had similar effects to traditional peripheral cues in two respects. First, it produced reliable orienting even when spatially uninformative (Experiments 1 and 2). Second, it still did so (at least, at relatively short SOAs) even when spatially counter-informative (Experiment 3 ). These similarities are noteworthy because the effects of peripheral cues have been attributed by numerous authors to reflexive, subcortical neural pathways involving the superior colliculus (e.g. Rafal et al., 1991; Rafal \& Henik, 1994; Spence \& Driver, 1994, 1997). Such neuroanatomical conclusions have been based on a wide range of data, including studies of patient groups with subcortical damage (see Rafal \& Henik, 1994), anatomical manipulations in healthy people (see Rafal et al., 1991) and analogies with animal studies (Spence \& Driver, 1997; Stein \& Meredith, 1993). It should be of considerable interest to study the neural basis for the orienting in response to seen gaze which we have identified here. It may seem unlikely that subcortical structures such as the superior colliculus could be involved, given their limited visual resolution 
for details of shape, such as those presumably required to perceive gaze direction. On the other hand, some limited face-perception abilities have recently been ascribed to subcortical structures, including the colliculus (e.g. Johnson \& Morton, 1991). Moreover, it is entirely possible that cortical structures specializing in gaze perception (e.g. cells in the superior temporal sulcus which appear tuned to gaze direction; see Perrett \& Emery, 1994; Perrett et al., 1990) could modulate subcortical structures that specialize in reflexive spatial orienting, and might do so in a relatively automatic fashion.

The present cueing effects from seen gaze also differed from those typically produced by uninformative peripheral cues, in two respects. First, their time-course was relatively delayed and extended. The effects of seen gaze were not apparent at the shortest SOA we used (for targets that followed the gaze cue by $100 \mathrm{msec}$, at which time standard peripheral cueing effects are usually apparent). The gaze effects emerged at $300 \mathrm{msec}$, and lasted at least $700 \mathrm{msec}$ when the gaze-cue was spatially uninformative (Experiment 2). In this respect, the effects of our uninformative gaze-cue resemble those of traditional informative central cues more closely than those caused by uninformative peripheral cues. The same applies to the absence of any IOR at the longer SOA following the gaze-cue, as discussed earlier. However, our gaze-cues differed in so many respects from standard central or peripheral cues (e.g. not only in their physical size and eccentricity, but also in the information that must be encoded to determine which side they should benefit), that further work would be needed for any full understanding of the basis for these similarities and dissimilarities.

We did not measure participants' own eye-movements during the present studies, and so cannot decisively judge whether covert or overt orienting mechanisms (or both) were responsible for the spatial cueing effects which we observed. Note that this issue does not undermine any of our specific conclusions concerning the automaticity of orienting in response to seen gaze. Moreover, although recent attention research has focused primarily on covert orienting (e.g. Posner, 1980; Spence \& Driver, 1994, 1996, 1997), it can be argued that overt orienting is of at least as much importance in everyday life. Nevertheless, in future research it would be useful to monitor for any saccades during our task, separating trials on which central fixation is maintained from those where overt orienting is observed. Our suspicion is that covert mechanisms were probably responsible for some of our effects, especially in Experiment 3, where we found that observers could not override their tendency to attend briefly in the direction a face looked, even when they knew that the target was four times as likely on the opposite side. It seems more likely to us that covert attention initially shifted in the direction of seen gaze in this study, rather than overt attention, since it is quite straightforward to demonstrate in everyday life that adults can suppress overt saccades in the direction of seen gaze, at will. 
Our experiments suggest that people apparently cannot suppress covert shifts of attention as readily.

The distinction between covert and overt attention is of considerable social significance, in addition to its interest for mainstream attention researchers. Many authors (e.g. Humphrey, 1976) have suggested that our ability to attend covertly in different directions may have evolved partly to mask our intentions and interests from others, who may be monitoring our gaze direction. Covert intentional mechanisms may allow us to "look" automatically where someone else is gazing, while disguising this fact from them and any other parties. An initial shift of covert attention may be sufficient to determine whether there is a peripheral event of sufficient import to merit a potentially revealing saccade.

In our studies, only the eyes of the central face deviated towards one side to provide the cueing event. As noted above when discussing naturalistic studies of shared attention in babies, spatial orienting may also be triggered by head turns and other seen changes in posture (e.g. pointing). The simple paradigm that we have developed here could be adapted to determine which visual properties of socially significant stimuli are most critical for triggering automatic orienting. In an independent study conducted at the same time as our own, Langton and Bruce (this issue) observed that the direction faced by a seen head can influence visual detection latencies for peripheral targets, with faster responses for targets in the direction (left, right, up or down) that a central head faced towards. This was observed in adult subjects even though the direction faced by the seen head was entirely unpredictive of target location, similar to our own findings for seen gaze in Experiments 1 and 2. Future studies could exploit the cueing method to examine how perceived head-direction and eye-direction jointly influence spatial orienting in the observer (e.g. when the seen head faces one way, and the seen eyes another: see Perrett \& Emery, 1994). Moreover, by modifying computerized face stimuli in various ways, it should be possible to determine exactly what visual properties of natural faces are critical for triggering attention shifts (e.g. Must two eyes be seen? Must they be seen in the context of a whole face? Are schematic eyes equally effective? Must the face be upright rather than inverted? Are head turns only effective for faces with open eyes?). A further study by Friesen and Kingstone (1998) has already shown that the gaze of highly schematic, cartoon faces can induce corresponding attention shifts in the observer, thus showing that simple configural properties of the eye region are sufficient to produce at least some of the spatial cueing effect.

As noted in the Introduction, various aspects of shared attention in social settings are abnormal in people with the neuropsychiatric disorder of autism (Baron-Cohen, 1989; Baron-Cohen et al., 1996a, b; Leekham et al., in press). This was one of the foundations for Baron-Cohen's (1995) proposal of specialized modules for the control of attention shifts in accordance with gaze 
perception. Specifically, he suggested that two modular mechanisms are involved in gaze-following. One mechanism (an eye direction detector) is responsible for the perception of other people's gaze direction. The second modular component (the shared attention mechanism) is responsible for executing shifts of the observer's own attention in the corresponding direction. Baron-Cohen's main reason for proposing two separate mechanisms to produce the kind of orienting that we have documented here was as follows. People with autism seem on the one hand to be impaired in shifting their own attention where others look (attributed to a malfunctioning shared attention mechanism); but on the other hand to be relatively preserved at making geometric judgements about the direction of perceived gaze (attributed by the model to an intact eye direction detector; see Leekham et al., in press). On this account, individuals with autism are not impaired on the more "mechanical" aspects of perceiving where others are looking, but only on the more "mentalistic" aspects of interpreting the direction of seen gaze as reflecting the internal mental state of another person; namely, where that person is likely to be attending.

It would be particularly interesting to apply the tests developed here to individuals with autism, or the related disorder of Asperger's syndrome (Frith, 1991), since the present studies reveal that orienting in the direction of seen gaze can arise in a fairly "mechanical" or automatic fashion even in normal individuals. Our adult subjects were fully aware of the spatial probabilities in our experiments, and of the fact that the central face was merely a computerized photograph, incapable of interacting with the peripheral letters in any way. Thus, the spatial cueing effects that we have identified are unlikely to have been caused by any mentalistic interpretations of the seen gaze, but rather by a mechanistic "reflex" to it. However, this reflexive response may only be present in those capable of interpreting gaze in the mentalistic manner that is appropriate for many social situations. For instance, the development of the reflex might be a necessary precursor of the ability for such mentalistic interpretation; or might even be a consequence of it. If either of these possibilities applies, the reflex should be absent in people with autism. These issues, like the others we have discussed, could all be resolved empirically by further extensions of the cueing methods developed here. For the present, we hope that our experiments illustrate that studies of social cognition might fruitfully exploit the methods of mainstream attention research, and that the latter research tradition could be enriched by further consideration of the many social functions of attention.

\section{REFERENCES}

Anstis, S.M., Mayhew, J.W., \& Motley, T. (1969). The perception of where a TV portrait is looking. American Journal of Psychology, 82, 427-489.

Argyle, M., \& Cook. M. (1976). Gaze and Mutual Gaze. Cambridge: Cambridge University Press. 
Baldwin, D. (1991). Infants' contribution to the achievement of joint reference. Child Development, 55, 1278-1289.

Bargh, J.A. (1992). The ecology of automaticity: Towards establishing the conditions needed to produce automatic processing effects. American Journal of Psychology, 105, 181-199.

Baron-Cohen, S. (1989). Joint attention deficit in autism: Towards a cognitive analysis. Development and Psychopathology, 1, 185-189.

Baron-Cohen, S. (1994). How to build a baby that reads minds: Cognitive mechanisms in mindreading. Cahiers de Psychologie Cognitive, 13, 513-552.

Baron-Cohen, S. (1995). Mindblindness: An essay on autism and theory of mind. Cambridge, MA: MIT Press.

Baron-Cohen, S., Allen, J., \& Gillberg, C. (1992). Can autism be detected at 18 months? The needle, the haystack, and the CHAT. British Journal of Psychiatry, 161, 166-180.

Baron-Cohen, S., Campbell, R., Karmiloff-Smith, A., Grant, J., \& Walker, J. (1996a). Are children with autism blind to the mental significance of the eyes? British Journal of Developmental Psychology.

Baron-Cohen, S., Cox, A., Barid, C., Swettenham, J., Drew, A., Nightingale, N., Morgan, K., \& Charman, T. (1996b). Psychological markers of autism at 18 months of age in a large population. British Journal of Psychiatry, 168, 158-163.

Bates, E., Benigni, L., Bretherton, L.I., Camioni, L., \& Volterra, V. (1979). Cognition and communication from 9-13 months: Correlational findings. In E. Bates (Ed.), The emergence of symbols: Cognition and communication in infancy. London: Academic Press.

Blest, A. (1957). The function of eyespot patterns in the Lepidoptera. Behavior, 11, 209-256.

Brothers, L. (1990). The social brain: A project for integrating primate behavior and neurophysiology in a new domain. Concepts in Neuroscience, 1, 27-51.

Brothers, L. (1995). The neurophysiology of the perception of intentions by primates. In M. Gazzaniga (Ed.), The cognitive neurosciences . Cambridge, MA: MIT Press.

Bruce, C., Desimone, R., \& Gross, C. (1981). Visual properties of neurons in a polysensory area in superior temporal sulcus. Journal of Neurophysiology, 46, 369-384.

Bruner, J. (1983). Child's talk: Learning to use language. Oxford: Oxford University Press.

Burghardt, G. (1990). Cognitive ethology and critical anthropomorphism: A snake with two heads and hog- nosed snakes that play dead. In C. Ristau (Ed.), Cognitive ethology: The minds of other animals. Hillsdale, NJ: Lawrence Erlbaum Associates Inc.

Butter, C., \& Snyder, D. (1972). Alterations in aversive and aggressive behaviors following orbital frontal lesions in rhesus monkeys. Acta Neurobiologica, 32, 523-565.

Butterworth, G. (1991). The ontogeny and phylogeny of joint visual attention. In A. White (Ed.), Natural theories of mind. Oxford: Blackwell.

Butterworth, G., \& Jarrett, N. (1991). What minds have in common is space: Spatial mechanisms serving joint visual attention in infancy. British Journal of Developmental Psychology, 9, 55-72.

Byrne, R., \& Whiten, A. (1991). Computation and mindreading in primate tactical deception. In A. White (Ed.), Natural theories of mind. Oxford: Blackwell.

Campbell, R., Heyward, C., Cowey, A., Regard, M., \& Landis, T. (1990). Sensitivity to gaze in prosopagnosic patients and monkeys with superior temporal sulcus ablation. Neuropsycholgia, 28, 1123-1142.

Carr, T.H. (1992). Automaticity and cognitive anatomy. American Journal of Psychology, 205, 201-237.

Chance, M. (1967). The interpretation of some agonistic postures: The role of "cut-off" acts and postures. Symposium of the Zoological Society of London, 8, 71-89.

Corkum, V., \& Moore, C. (1995). Development of joint visual attention in infants. In C. Moore \& P.J. Dunham (Eds) Joint attention: Its origins and role in development . Hillsdale, NJ: Lawrence ErIbaum Associates Inc. 
Damasio, A.R. (1995). Descartes' error: Emotion, reason and the human brain. London: Picador.

Enns, J.T., Ochs, E.P., \& Rensink, R.A. (1990). V-scope. Behaviour Research Instruments \& Computers, 22, 118-122

Fodor, J. (1983). The modularity of mind. Cambridge, MA: MIT Press.

Friesen, C.K., \& Kingstone, A. (1998). The eyes have it: Reflexive orienting is triggered by nonpredictive gaze. Psychonomic Bulletin and Review, 5, 490-493.

Frith, U. (1991). Autism and Asperger's syndrome. Cambridge: Cambridge University Press.

Gibson, J., \& Pick, A. (1962). Perception of another person's looking behavior. American Journal of Psychology, 76, 386-394.

Henik, A., Rafal, R.D., \& Rhodes, D. (1995). Endogenously generated and visual guided saccades after lesions of the human frontal eye fields. Journal of Cognitive Neuroscience, 6 , 400-411.

Hood, B.M., Willen, J.D., \& Driver, J. (1998). Gaze perception triggers corresponding shifts of visual attention in young infants. Psychological Science, 9, 131.

Humphrey, N. (1976). The social function of intellect. In P. Bateson \& R. Hinde (Eds), Growing Points in Ethology. Cambridge: Cambridge University Press.

Johnson, M.H., \& Morton, J. (1991). Biology and cognitive development: The case of face recognition. Oxford: Blackwell.

Jonides, J. (1981). Voluntary versus automatic control over the mind's eye's movements. In J.B. Long \& A.D. Baddeley (Eds), Attention and Performance IX. Hillsdale, NJ: Lawrence Erlbaum Association Inc.

Klein, R.M., Kingstone, A., \& Pontefract, A. (1992). Orienting of visual attention. In K. Rayner (Ed.), Eye-movements and visual cognition: Scene perception and reading. New York: Springer-Verlag.

Klein, R.M., \& Taylor, T.L. (1994). Categories of cognitive inhibition with reference to attention. In T.H. Carr \& D. Dagenbach (Eds), Inhibitory processes in attention memory, and language. San Diego, CA: Academic Press.

Kling, A., \& Brothers, L. (1992). The amygdala and social behavior. In J. Aggleton (Ed.), Neurobiological aspects of emotion, memory and mental dysfunction. New York: Wiley.

Kluver, H., \& Bucy, P. (1938). An analysis of certain effects of bilateral temporal lobectomy in the rhesus monkey, with special reference to "psychic blindness". Journal of Psychology, 5, 33-54.

Lambert, A., \& Sumich, A.L. (1996). Spatial orienting controlled without awareness: A semantically based implicit learning effect. Quarterly Journal of Experimental Psychology, 49A, 490-518.

Langton, S.R.H., \& Bruce, V. (this issue). Reflexive visual orienting in response to the social attention of others. Visual Cognition, 6, 541-567.

Leekham, S., Baron-Cohen, S., Brow, S., Perrett, D., \& Milders, M. (in press). Eye-direction detection: A dissociation between geometric and joint-attention skills in autism. British Journal of Developmental Psychology.

Logan, G.D. (1992). Attention and preattention in theories of automaticity. American Journal of Psychology, 105, 317-339.

Lupianez, J., Milan, E.G., Tornay, F.J., Madrid, E., \& Tudelo, P. (1997). Does IOR occur in discrimination tasks? Yes, it does, but later. Perception \& Psychophysics, 59, 1241-1254.

Maurer, D. (1985). Infants perception of facedness. In T. Field \& M. Fox (Eds), Social Perception in Infants. Norwood, NJ: Ablex.

Mendelsohn, M., Haith M., \& Goldman-Rakic, P. (1982). Face scanning and responsiveness to social cues in infant monkeys. Developmental Psychology, 18, 222-228.

Menzel, E., \& Halperin, S. (1975). Purposive behavior as a basis for objective between chimpanzees. Science, 189, 652-654. 
Miller, J. (1988). A warning about median reaction time. Journal of Experimental Psychology: Human Perception and Performance, 14, 539-543.

Moore, C., \& Dunham, P. (1994). Joint attention: Its origins and role in development Hillsdale, NJ: Lawrence Erlbaum Associates Inc.

Muir, D., Hains, S., Cao, Y., \& D’Entremont, B. (1996). 3- to 6-month-olds' sensitivity to adult intentionality: The role of adult contingency and eye direction in dyadic interactions. Infant Behavior and Development, 19.

Muller, H.J., \& Rabbit, P.M.A. (1989). Reflexive and voluntary orienting of visual attention: Timecourse of activation and resistance to interruption. Journal of Experimental Psychology: Human Perception and Performance, 15, 315-330.

Papousek, H., \& Papousek, M. (1979). Early ontogeny of human social interaction: Its biological roots and social dimensions. In M. von Cranach, K. Foppa, W. Lepenies, \& D. Ploog (Eds), Human ethology: Claims and limits of a new discipline. Cambridge: Cambridge University Press.

Perrett, D., \& Emery, N.J. (1994). Understanding the intentions of others from visual signals: Neuropsychological evidence. Cahiers de Psychologie Cognitive, 13, 683-694.

Perrett, D., Harries, M., Mistlin, A., Hietanen, J, Benson, P., Bevan, R., Thomas, S., Oram, M., Ortega, J., \& Brierley, K. (1990). Social signals analysed at the single cell level: Someone is looking at me, something touched me, something moved! International Journal of Comparative Psychology, 4, 25-55.

Perrett, D., \& Mistlin, A. (1990). Perception of facial characteristics by monkeys. In W. Stebbins \& M. Berkely (Eds), Vol. 2. New York: Wiley.

Perrett, D., Rolls, E., \& Cann, W. (1982). Visual neurones responsive to faces in the monkey temporal cortex. Experimental Brain Research, 47, 329-342.

Posner, M.I. (1978). Chronometric explorations of mind. Hillsdale, NJ: Lawrence Erlbaum Associates Inc.

Posner, M.I. (1980). Orienting of attention. Quarterly Journal of Experimental Psychology, $32 A, 3-25$.

Posner, M.I., \& Cohen, Y. (1984). Components of visual orienting. In H. Bouma \& D.G. Bowhuis (Eds), Attention and performance X. Hove, UK: Lawrence Erlbaum Associates Ltd.

Premack, D. (1988). "Does the chimpanzee have a theory of mind?" revisited. In R. Byrne \& A. Whiten (Eds), Machiavellian Intelligence: Social expertise and the evolution of intellect. Oxford: Oxford University Press.

Rafal, R.D., \& Henik, A. (1994). The neurology of inhibition: Integrating controlled and automatic processes. In D. Dagenbach \& T.H. Carr (Eds), Inhibitory processes and attention, memory and language. London: Academic Press.

Rafal, R.D., Henik, A., \& Smith, J. (1991). Extrageniculate contributions to reflex visual orienting in normal humans: A temporal hemifield advantage. Journal of Cognitive Neuroscience, 3, 322-328.

Ristau, C. (1990). Aspects of the cognitive ethology of an injury feigning plover. In C. Ristau (Ed.), Cognitive ethology: The minds of other animals. Hillsdale, NJ: Lawrence Erlbaum Associates Inc.

Rubin, A. (1970). Measurement of romantic love. Journal of Personal and Social Psychology, 16, 265-273.

Sagi, D., \& Julesz, B. (1985). What and where in vision. Science, 228, 1217-1219.

Samuels, C. (1985). Attention to eye contact opportunity and facial motion by 3 month old infants. Journal of Experimental Child Psychology, 40, 105-114.

Scaife, M. (1976). The response to eye-like shapes by birds. II. The importance of staring, pairedness, and shape. Animal Behavior, 24, 200-206.

Scaife, M., \& Bruner, J. (1975). The capacity for joint visual attention in the infant. Nature, 253, $265-266$. 
Shiffrin, R.M., \& Schneider, W. (1977). Controlled and automatic human information processing: II. Perceptual learning, automatic attending, and a general theory. Psychological Review, 84, 127-190.

Simon, J.R., \& Craft, J.L. (1970). Effects of an irrelevant auditory stimulus on visual choice reaction time. Journal of Experimental Psychology, 86, 272-274

Spence, C.J., \& Driver, J. (1994). Covert spatial orienting in audition: Exogenous and endogenous mechanisms. Journal of Experimental Psychology: Human. Perception and Performance, 20, 555-574.

Spence, C.J., \& Driver, J. (1996). Audiovisual links in endogenous covert spatial attention. Journal of Experimental Psychology: Human Perception and Performance, 22, 1005-1030.

Spence, C.L, \& Driver, J. (1997). Audiovisual links in exogenous covert spatial attention. Perception and Psychophysics, 59, 1-22.

Stein, B.E., \& Meredith, M.A. (1993). The merging of the senses. Cambridge, MA: MIT Press.

Treisman, A.M., \& Gelade, G. (1980). A feature integration theory of attention. Cognitive Psychology, 12, 97-136.

Van Hooff, J. (1962). Facial expressions in higher primates. Symposium of the Zoological Society of London, 8, 97-125.

Vecera, S., \& Johnson, M.J. (1995). Gaze detection and the cortical processing of faces in infants. Visual Cognition, 2, 59-87.

Watt, R.J. (1992). Faces and vision. In V. Bruce \& M. Burton (Eds), Processing images of faces. Norwood, NJ: Ablex.

Wolff, P. (1963). Observations on the early development of smiling. In B. Foss (Ed.), Determinants of Infant Behavior, Vol. 2. New York: Wiley.

Manuscript received October 1997 Revised manuscript received April 1998 